\title{
Apoio matricial em saúde mental na atenção primária à saúde:
}

\author{
uma análise da produção científica e documental
}

Iris Guilherme Bonfim ${ }^{1}$ Evelyne Nunes Ervedosa Bastos ${ }^{2}$

Cezar Wagner de Lima Góis ${ }^{3}$ Luis Fernando Tófoli ${ }^{4}$

BONFIM, I.G. et al. Matrix support in mental health in primary halth care: a review of scientific papers and official documents. Interface (Botucatu), v.17, n.45, p.287-300, abr./jun. 2013.

The challenge of an effective comprehensive care in the Brazilian National Health System sustains a debate about the mMatrix support (MS) for the Family Health Strategy (FHS) while stressing the need for further studies on the subject. This paper aims to critically analyze the matrix support with the aid of scientific papers and documents from the Brazilian Ministry of Health, especially those directed to mental health $(\mathrm{MH})$ matrix support in primary care. The results emphasize the importance of coordination between the $\mathrm{MH}$ and primary care, though they also indicate that $\mathrm{MS}$ in $\mathrm{MH}$ is encompassed within a health care model that is still under construction and that new theoretical-practical contributions and evaluative studies are needed for its improvement.

Keywords: Matrix support. Mental health. Primary health care. Family health strategy.
O desafio da efetivação da integralidade da atenção no Sistema Único de Saúde (SUS) favorece a discussão sobre o apoio matricial (AM) para a Estratégia Saúde da Família (ESF) e evidencia a necessidade de mais estudos sobre o tema. Neste artigo analisa-se criticamente o apoio matricial por meio de publicações em periódicos e documentos do Ministério da Saúde, especialmente aqueles focados no apoio matricial em saúde mental $(S M)$ na atenção primária. $\mathrm{O}$ material estudado enfatiza a importância da articulação entre a SM e a atenção primária, embora também indique que o AM em SM faz parte de um modelo de atenção à saúde em construção e novos aportes teórico-práticos e estudos avaliativos são indispensáveis para seu aperfeiçoamento.

Palavras-chave: Apoio matricial. Saúde mental. Atenção primária à saúde. Estratégia Saúde da Família.

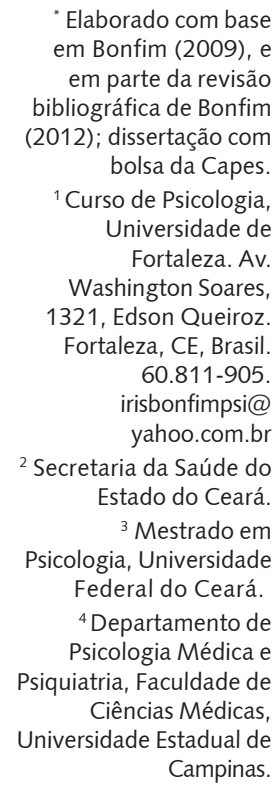
bolsa da Capes.

${ }^{1}$ Curso de Psicologia Universidade de Fortaleza. Av. Washington Soares, 1321, Edson Queiroz. Fortaleza, CE, Brasil. 60.811-905. irisbonfimpsi@ yahoo.com.br 2 Secretaria da Saúde do Estado do Ceará. ${ }^{3}$ Mestrado em Psicologia, Universidade Federal do Ceará. ${ }^{4}$ Departamento de Psicologia Médica e Psiquiatria, Faculdade de Ciências Médicas Universidade Estadual de Campinas. 


\section{Introdução}

Apesar de um considerável progresso obtido desde a Declaração de Alma-Ata (Opas, 1978), os sistemas de saúde ainda estão organizados para a atenção às doenças agudas. Assim, continua sendo um desafio empreender cuidados inovadores para dar conta, também, das condições crônicas de saúde - incluindo as doenças mentais.

Neste sentido, o Brasil - por meio do Sistema Único de Saúde (SUS) - vem construindo estratégias possíveis de contribuir para a superação desta limitação. Podemos destacar, entre estas estratégias: o estabelecimento da Estratégia Saúde da Família (ESF) como eixo organizador da Atenção Primária à Saúde (APS); a Política de Saúde Mental com a reversão do modelo centrado no hospital psiquiátrico e com a criação de uma rede de serviços substitutivos a este; a Política Nacional de Humanização e as estratégias de mudança no sistema vinculadas a esta; e a Política Nacional de Educação Permanente. Entretanto, apesar de todo o esforço e dos significativos avanços, estes ainda são modestos diante dos desafios impostos pela realidade sanitária aos profissionais e serviços de saúde e à população (Góis, 2008).

Assim, persistem, no SUS, as tradicionais formas de funcionamento dos serviços de saúde, que mantêm processos de trabalho fragmentados, centrados em procedimentos e com foco de atuação no modelo biomédico hegemônico, que dificultam o trabalho em equipe e a integração dos saberes de várias categorias profissionais, que poderiam facilitar a concretização do princípio da integralidade com maior potência para o SUS (Campos, 1999, 1998).

Seria, portanto, uma tarefa de o processo reflexivo sobre a saúde coletiva compreender melhor os modelos que estruturam os serviços e sistemas de saúde. Segundo Paim (1994), modelos assistenciais são:

[...] combinações tecnológicas utilizadas pela organização dos serviços de saúde em determinados espaços-populações, incluindo ações sobre o ambiente, grupos populacionais, equipamentos comunitários e usuários de diferentes unidades prestadoras de serviços de saúde com distinta complexidade. (Paim, 1994, p.457)

De forma complementar, Campos (1989, p.53) define os modelos assistenciais como "o modo como são produzidas as ações de saúde e a maneira como os serviços de saúde e o Estado se organizam para produzi-las e para distribuí-las". Posteriormente, o conceito de modelos de atenção veio ampliar e superar a perspectiva técnico-assistencial, incluindo, além de um componente técnico (também chamado de operativo), as vertentes gerencial e organizativa (Teixeira, 2006). Conforme Mendes (2010), as redes de atenção à saúde são compostas por três elementos constituintes: a população, a estrutura operacional e os modelos de atenção. Para Mendes, estes elementos são:

Sistemas lógicos que organizam o funcionamento das redes de atenção à saúde, articulando, de forma singular, as relações entre a população e suas subpopulações estratificadas por riscos, os focos das intervenções do sistema de atenção à saúde e os diferentes tipos de intervenções sanitárias, definidos em função da visão prevalecente da saúde, das situações demográfica e epidemiológica e dos determinantes sociais da saúde, vigentes em determinado tempo e em determinada sociedade. (Mendes, 2010, p.2302)

É lícito, pois, a partir deste ponto, lançar a questão sobre quais as formas de organização das redes e modelos de atenção que poderiam mudar uma estrutura de saúde centrada na doença e na figura do médico, com um trabalho frequentemente fragmentado e avesso à colaboração.

No intuito de contribuir com a confrontação de um modelo de atenção burocratizado e centrado na doença, Campos $(1999,1998)$ propõe o Apoio Matricial (AM) como uma estratégia de organização do trabalho em saúde cuja atuação deve ser complementar aos sistemas de referência e contrarreferência e às centrais de regulação da atenção especializada. Desta forma, o AM sugere uma mudança radical na conduta do especialista, indicando que este passe a ter uma postura dialógica e horizontal com os outros profissionais da rede de saúde. 
O AM objetiva a oferta de suporte assistencial e técnico-pedagógico, pelo especialista, para equipes interdisciplinares que atuem como equipes de referência e possuam uma clientela adscrita. Essas equipes de referência buscam ampliar a possibilidade de construção de vínculo entre profissionais e usuários e reforçar o poder de gestão da equipe interdisciplinar (Campos, Domitti, 2007).

O apoiador matricial é um profissional especializado em alguma área de conhecimento (saúde mental, educação física, nutrição, fisioterapia etc.) que difere da área de conhecimento da equipe de referência, e que pode apoiar esses profissionais com determinadas informações e intervenções voltadas para contribuir na ampliação da resolutividade das ações dessa equipe.

Como evidenciado, a proposta do AM tem sido incentivada pelo Ministério da Saúde (MS) nas Políticas Nacionais de Saúde Mental, de Atenção Básica e de Humanização (Campos, Domitti, 2007). Esta pode ser considerada uma estratégia inovadora (OMS, 2003) para atenção às condições crônicas de saúde na APS brasileira (Onocko Campos et al., 2012).

Desde 2001, em oficinas de trabalho e congressos, o MS já demonstrava interesse na aproximação entre a saúde mental e a atenção primária (Onocko Campos, Gama, 2008). Em 2003, o MS apontou o apoio matricial como diretriz para a inclusão das ações de saúde mental na atenção primária (Brasil, 2003). No entanto, o incentivo financeiro para a sua realização só aconteceu com a criação do Núcleo de Apoio à Saúde da Família (NASF) (Brasil, 2008).

Conforme determina a portaria que criou o NASF, os núcleos devem ser constituídos por equipes formadas por profissionais de diferentes áreas do conhecimento, atuando de forma compartilhada e dividindo, com as equipes de saúde da família, a responsabilidade pelas ações de saúde no território onde atuam. Além disso, esses profissionais precisam, segundo a portaria, rever as práticas de encaminhamento baseadas nos processos de referência e contrarreferência. O objetivo do NASF é qualificar as ações da ESF, bem como ampliar a resolutividade e a integralidade da APS e, consequentemente, do SUS.

De acordo com o Ministério da Saúde (Brasil, 2009), o apoio matricial constitui uma das ferramentas necessárias para a organização do processo de trabalho do NASF e evidencia este núcleo como uma das principais estratégias de atenção em saúde mental na atenção primária nos últimos anos (Brasil, 2011) o que favorece a realização do apoio matricial em saúde mental para a ESF e fortalece a necessidade de mais estudos sobre este.

Os autores deste trabalho ancoraram seus questionamentos em busca de maior compreensão do apoio matricial em saúde mental a partir das suas experiências na implantação desta modalidade de atenção na cidade de Fortaleza-CE. Em 2005, Fortaleza iniciou um redirecionamento políticoorganizacional na Secretaria Municipal de Saúde (SMS) que estabeleceu a saúde mental e a atenção primária entre as áreas prioritárias de investimento. Isto levou a um processo gradual de implantação do AM em SM no município a partir de 2006, intensificado no biênio 2008-2009, mas ainda não totalmente estabelecido até o presente momento.

Diante da nossa inquietação no contexto da prática, decidimos investigar de forma sistemática a produção teórico-conceitual a respeito desse arranjo organizacional (Bonfim, 2009). Assim, o objetivo deste artigo é analisar criticamente o apoio matricial por meio das publicações em periódicos científicos e documentos do Ministério da Saúde, enfocando, de modo especial, o apoio matricial em saúde mental na atenção primária.

\section{Metodologia}

Este artigo consiste em um estudo de natureza qualitativa do tipo exploratório, incluindo um levantamento bibliográfico (Gil, 1999) e uma pesquisa documental (Leopardi, 2002), sobre a construção do conhecimento acerca do apoio matricial.

Inicialmente, fizemos um levantamento bibliográfico, na Biblioteca Virtual da Saúde (BVS), dos artigos científicos que apresentavam os termos "apoio matricial", "apoio matricial em saúde mental" e "matriciamento em saúde mental", referentes aos anos de 1998 a fevereiro de 2012 (não há descritores específicos para apoio matricial). Os autores selecionaram dois grupos de artigos científicos: 1) aqueles 
com reflexões teóricas e práticas sobre apoio matricial sem fazer referência específica a uma especialidade de atenção à saúde, e 2) aqueles focados no apoio matricial em saúde mental na APS.

Desta forma, foi encontrado, na BVS, um total de 26 artigos científicos que se encaixavam nestes perfis.

Entretanto, no decorrer da análise do material bibliográfico, foram incluídos mais sete artigos publicados em periódicos científicos e livros sobre saúde coletiva que se adequavam ao perfil dessa revisão, com vistas a enriquecer a revisão bibliográfica sobre o tema.

Foram analisados, ainda, documentos do Ministério da Saúde (Brasil, 2011, 2009, 2008, 2007, 2005 , 2004a, 2004b, 2004c, 2003) que abordaram o tema apoio matricial entre 2003 e fevereiro de 2012.

A etapa seguinte iniciou-se com a leitura dos artigos e documentos num exercício de compreenderIhes o conteúdo central. Posteriormente, realizamos uma leitura mais detalhada e qualificada para a elaboração de uma análise crítica sobre os artigos e documentos. Depois, fizemos o fichamento destes, organizando e classificando cuidadosamente as principais ideias dos autores e dos documentos. A partir disso, duas grandes categorias emergiram: Diretrizes do Apoio Matricial e Apoio Matricial em Saúde Mental na Atenção Primária, a respeito das quais discorreremos a seguir.

\section{Discussão}

Com a análise do ano de publicação dos artigos utilizados nesta pesquisa, observamos um grande intervalo de tempo - oito anos - entre a publicação do segundo artigo que define o apoio matricial em saúde (Campos, 1999) e os posteriores. Isso pode ter se dado pelo fato de que não havia financiamento específico para estimular o apoio matricial, limitando sua implantação em municípios onde os gestores fossem sensíveis a essa metodologia. Tal fato também pode justificar a escassa produção científica sobre o tema nesse período. A partir de 2003, vários documentos institucionais do Ministério da Saúde (Brasil, 2007, 2005, 2004a, 2004b, 2004c, 2003) trazem a proposta do apoio matricial. Apesar disso, é somente com a criação do NASF (Brasil, 2008) que o Ministério da Saúde possibilita o financiamento que estimula a ação de apoio matricial na ESF.

No Quadro 1, consta a temática central de cada artigo selecionado, o número de itens e a referência dos artigos ligados a cada tema. No Quadro 2, a descrição, a quantificação e as referências dos documentos ministeriais selecionados. Cabe ressaltar: os documentos oficiais que mencionam ou se referem ao apoio matricial foram emitidos somente a partir do ano de 2003.

Examinemos, agora, as duas categorias centrais da análise realizada por este artigo.

\section{Diretrizes do apoio matricial}

Para a organização do trabalho em apoio matricial e equipe de referência, há desafios tanto estruturais quanto organizacionais. Os obstáculos estruturais, decorrentes do excesso de demanda e carência de recursos, são uma evidência do distanciamento entre as diretrizes políticas dos documentos oficiais do Estado brasileiro e a execução e cobertura do SUS na realidade dos municípios.

A existência, porém, dos problemas estruturais não exclui a existência de obstáculos de ordem organizacional (Campos, Domitti, 2007), que Campos e Cunha (2011) reconhecem como dependentes, também, dos obstáculos estruturais. Entretanto, na ótica de Campos e Cunha, a superação dos obstáculos organizacionais pode ser, em parte, implementada por gestores e profissionais.

Assim, a organização tradicional na forma gerencial hegemônica dos serviços de saúde contribui para gerar uma série de consequências indesejáveis: alienação do trabalhador e limitação do olhar dos profissionais sobre o processo saúde-doença; concentração da atenção apenas na enfermidade e em procedimentos; restrição da clínica à tradicional terapêutica com fármacos (Campos, Rattes, 2008); e desvalorização do saber de outras áreas de conhecimento, fundamentais para uma atenção integral ao usuário. Diante desse contexto, as diretrizes do apoio matricial (Campos, 1999) focam-se em esforços significativos para sua organização, os quais podem contribuir para reduzir a potência de alguns obstáculos organizacionais. 
Quadro 1. Temática central, quantidade e referências dos artigos analisados por cada temática.

\begin{tabular}{|c|c|c|}
\hline Temática abordada nos artigos científicos & $\begin{array}{l}\mathrm{N}^{\circ} \mathrm{de} \\
\text { Itens }\end{array}$ & Artigos que se referem à temática apontada \\
\hline \multicolumn{3}{|l|}{ Reflexões teórico-conceituais } \\
\hline $\begin{array}{l}\text { Apoio matricial } \\
\text { Apoio matricial em saúde mental na APS } \\
\text { Apoio matricial + NASF }\end{array}$ & $\begin{array}{l}5 \\
2 \\
1\end{array}$ & $\begin{array}{l}\text { Campos, 1998, 1999; Campos, Rates, 2008; Oliveira, } \\
\text { 2008; Campos, Domitti, } 2007 . \\
\text { Onocko Campos, Gama, 2008; Figueiredo, Onocko } \\
\text { Campos, } 2008 . \\
\text { Campos, Cunha, 2011. }\end{array}$ \\
\hline \multicolumn{3}{|l|}{$\begin{array}{l}\text { Reflexões teórico-conceituais e pesquisas qualitativas } \\
\text { sobre saúde mental na APS que enfocam o apoio } \\
\text { matricial em saúde mental }\end{array}$} \\
\hline $\begin{array}{l}\text { Pesquisas avaliativas com usuários e profissionais } \\
\text { Pesquisa com conselheiros de saúde } \\
\text { Pesquisa com profissionais de CAPS } \\
\text { Pesquisa com profissionais da ESF } \\
\text { Pesquisa com profissionais de CAPS e da ESF } \\
\text { Pesquisa com coordenadores de CAPS infantil e } \\
\text { unidades de saúde da família } \\
\text { Pesquisa com profissionais de SM, da ESF e com } \\
\text { gestores }\end{array}$ & $\begin{array}{l}2 \\
1 \\
1 \\
4 \\
1 \\
1 \\
1 \\
1\end{array}$ & $\begin{array}{l}\text { Onocko Campos et al., 2012, } 2011 . \\
\text { Conssetin, OlschowsKy, } 2011 . \\
\text { Bezerra, Dimenstein, 2008. } \\
\text { Cavalcante et al., 2011; Milke, Olschowsky, 2010; } \\
\text { Dimenstein et al., 2009a, 2009b. } \\
\text { Souza et al., 2011. } \\
\text { Delfini, Reis, } 2012 . \\
\text { Figueiredo, Onocko Campos, } 2009 .\end{array}$ \\
\hline \multicolumn{3}{|l|}{ Pesquisa Intervenção } \\
\hline $\begin{array}{l}\text { Realizada por estudantes de Residência } \\
\text { Multiprofissional com agentes comunitários de } \\
\text { saúde }\end{array}$ & 1 & Carneiro et al., 2009. \\
\hline \multicolumn{3}{|l|}{$\begin{array}{l}\text { Relatos de experiência que enfocam apoio matricial } \\
\text { em saúde mental na atenção primária à saúde }\end{array}$} \\
\hline $\begin{array}{l}\text { Apoio matricial em saúde mental na APS } \\
+ \text { Outras especialidades } \\
+ \text { NASF } \\
+ \text { Residência Multiprofissional }\end{array}$ & $\begin{array}{l}5 \\
1 \\
1 \\
1\end{array}$ & $\begin{array}{l}\text { Prestes et al., 2011; Delfini et al., 2009; Soares, } \\
\text { 2008; Bardan, Oliveira, 2007; Tófoli, Fortes, } 2007 . \\
\text { Arona, 2009. } \\
\text { Bezerra et al., } 2010 . \\
\text { Meira, Silva, } 2011 .\end{array}$ \\
\hline \multicolumn{3}{|l|}{$\begin{array}{l}\text { Ponderações sobre apoio matricial em saúde mental na } \\
\text { APS e pesquisas documentais }\end{array}$} \\
\hline $\begin{array}{l}\text { Abordam o profissional de psicologia e sua incursão } \\
\text { em políticas públicas de atenção primária e de saúde } \\
\text { mental }\end{array}$ & 2 & Böing, Crepald, 2010; Ferreira Neto, 2008. \\
\hline \multicolumn{3}{|l|}{$\begin{array}{l}\text { Pesquisa com agentes comunitários de saúde sobre } \\
\text { Depressão }\end{array}$} \\
\hline $\begin{array}{l}\text { Apoio matricial em saúde mental apontado como } \\
\text { ferramenta determinante para a qualidade do } \\
\text { atendimento às pessoas com transtornos mentais na } \\
\text { atenção primária }\end{array}$ & 1 & Harada, Soares, 2010. \\
\hline \multicolumn{3}{|l|}{ Estudo de caso } \\
\hline $\begin{array}{l}\text { Sobre um usuário acompanhado no apoio matricial } \\
\text { em saúde mental em um Centro de Saúde da Família }\end{array}$ & 1 & Vieira Filho, Rosa, 2011. \\
\hline Total de itens & 32 & \\
\hline
\end{tabular}


Quadro 2. Descrição, quantidade e referências dos documentos analisados

\begin{tabular}{|l|c|l|}
\hline \multicolumn{1}{|c|}{ Documentos oficiais } & No de Itens & Referência dos documentos \\
\hline Legislação & 1 & Brasil, 2008. \\
\hline Circular Conjunta - Coord. de Saúde Mental e Coord. da Atenção Primária & 1 & Brasil, 2003. \\
\hline Documento da Política Nacional de Atenção Primária & 1 & Brasil, 2009. \\
\hline Documentos da Política Nacional de Saúde Mental & 2 & Brasil, 2005, 2004b. \\
\hline Cartilhas da Política Nacional de Humanização & 2 & Brasil, 2004a, 2004c. \\
\hline Relatórios de Gestão da Coordenação Nacional de Saúde Mental & 2 & Brasil, 2007, 2011. \\
\hline Total de itens & 9 & \\
\hline
\end{tabular}

A primeira delas é a diretriz de gestão colegiada, que aponta para a necessidade da existência de espaços coletivos para a discussão e reflexão sobre os processos de trabalho (Figueiredo, Onocko Campos, 2009, 2008; Oliveira, 2008). Ela demonstra, assim, a importância da gestão compartilhada entre trabalhadores e gestores, com algum grau de democracia institucional.

Desta forma, a organização do processo de trabalho em apoio matricial e equipe de referência depende da efetivação de projetos coletivos pactuados entre a gestão e os profissionais (Arona, 2009; Dimenstein et al., 2009a), exigindo-Ihes autonomia e compromisso para a efetivação dos processos de trabalho pactuados coletivamente.

A segunda diretriz, vínculo terapêutico, apontada por Campos (1999), sobretudo, a partir da equipe de referência, tem o objetivo de realizar um acompanhamento mais próximo dos usuários sob seus cuidados. Contribui, assim, para a avaliação das necessidades de saúde dos usuários e para o estímulo de uma relação de confiança e de vínculo terapêutico entre profissionais e usuários, mesmo que estes estejam sendo atendidos também em outros serviços.

Entretanto, conforme demonstra a análise de outras produções que abordam o apoio matricial (Delfini, Reis, 2012; Mielke, Olchowshy, 2010; Brasil, 2009; Carneiro et al., 2009), a construção de vínculo deve ser estimulada não somente na relação entre profissionais e usuários, mas, também, entre os próprios profissionais. Isto favorece a integração da equipe, facilita a troca de informações e de afetos entre eles, possibilita um ambiente mais prazeroso para profissionais e usuários, e mais rico em conhecimentos e em sentimentos positivos.

A terceira diretriz é a de interdisciplinaridade das práticas e dos saberes. Para um esclarecimento mais detalhado dessa diretriz, fundamental para a efetivação do apoio matricial, Campos e Cunha (2011) afirmam a necessidade de trabalhar com uma ferramenta conceitual que contribui para promover uma mudança epistêmica de flexibilização das rígidas fronteiras entre as profissões de saúde. Tal ferramenta corresponde aos conceitos de núcleo e campo de conhecimento.

O núcleo corresponde ao tradicional conjunto de conhecimentos e ações que são específicos de determinada categoria profissional. Por sua vez, o conceito de campo representa uma abertura da identidade profissional cristalizada para uma atuação voltada à interdisciplinaridade e à interprofissionalidade. Desta forma, o conceito de campo corresponde ao conhecimento a ser apropriado por profissionais que atuam em um mesmo contexto, com vistas a conseguirem imprimir eficácia e eficiência ao trabalho (Campos, Cunha, 2011).

Sendo assim, conforme a diretriz de interdisciplinaridade das práticas e dos saberes, a organização dos arranjos apoio matricial e equipe de referência colabora para a troca de saberes, favorece a comunicação ativa e o estabelecimento de relações horizontais entre profissionais de distintos núcleos de conhecimentos (Oliveira, 2008). Ajuda, também, na transformação do modelo biomédico hegemônico por meio da ampliação da clínica (Brasil, 2004a, 2004c).

A um apoiador matricial, impõem-se determinados desafios. Entre estes, a interação com outros profissionais de saúde e a realidade complexa da maioria dos usuários que são atendidos no SUS. O 
saber de cada núcleo profissional jamais dará conta totalmente da complexidade do processo saúdedoença. Frequentemente, o apoiador será interpelado por esta complexidade e pela interação entre as profissões nesta estratégia de organização do trabalho em saúde. Para a garantia da integralidade da atenção, bem como para a ampliação da resolutividade do SUS, faz-se indispensável a construção interdisciplinar.

Desta forma, a proposta desses dois arranjos exige mudanças não só na estrutura organizacional dos serviços, mas, também, nas atitudes e práticas de profissionais e de gestores, buscando uma renovação cultural dos serviços e dos profissionais e gestores da saúde (Oliveira, 2008).

$\mathrm{Na}$ implementação do apoio matricial, inúmeros desafios são colocados para gestores e profissionais da ESF e do NASF pelo país. Em relação aos gestores, enquanto muitos reconhecem a necessidade desse arranjo, outros o implementam, exclusivamente, pelo estímulo do financiamento do NASF. Contudo, esse mesmo reconhecimento parece ser raro no que se refere ao estímulo à reorganização das ações de saúde, a partir das diretrizes sugeridas pelo Ministério e pelas publicações sobre o tema.

\section{Apoio Matricial em Saúde Mental na Atenção Primária}

Os artigos analisados trazem várias discussões relacionadas ao apoio matricial em saúde mental na APS em diferentes cidades do Brasil: Campinas-SP, São Paulo-SP, Fortaleza-CE, Camaragibe-PE, SalvadorBA, Belo Horizonte-MG, Sobral-CE, São José do Rio Preto-SP, Capivari-SP, Palmas-TO e Natal-RN. Destacaremos, a seguir, as principais contribuições encontradas no escopo destes artigos e nos documentos do MS, com base em duas subcategorias: Integração entre saúde mental e APS e Desafios e potencialidades do apoio matricial e do NASF na saúde mental.

\section{Integração entre saúde mental e atenção primária à saúde}

Diversos autores (Prestes et al., 2011; Sousa et al., 2011; Delfini et al., 2009; Dimenstein et al., 2009a, 2009b; Figueiredo, Onocko Campos, 2009, 2008; Bezerra, Dimenstein, 2008) apontam a necessidade de avanços na Reforma Psiquiátrica brasileira, destacando a necessidade de interligação entre redes assistenciais de saúde mental e de APS para favorecer um cuidado integral ao usuário.

Houve, de forma paralela, avanços nas redes de atenção primária à saúde, consolidada através da ESF, e da saúde mental, com a criação dos serviços substitutivos ao hospital psiquiátrico, sobretudo dos serviços tipo CAPS. Contudo, ainda há pouca integração entre as redes de cuidados de saúde mental e da APS. Esta limitação traz sérias consequências, tanto para o SUS, como um sistema unificado e integral, quanto para a APS e para a saúde mental (Onocko Campos et al., 2012, 2011; Figueiredo, Onocko Campos, 2008).

Desta forma, evidenciam-se vários motivos para a relevância da integração da saúde mental na APS: a alta prevalência de transtornos mentais; a ampliação do acesso aos cuidados de saúde mental; a ampliação da integralidade na saúde, reconhecendo a indissociabilidade entre os problemas de saúde mental e os "físicos"; o aumento da qualidade das ações e dos serviços de saúde (Brasil, 2009); entre outros.

Ademais, a ESF pode ser considerada um recurso estratégico para trabalhar com pessoas em sofrimento psíquico, em virtude da proximidade e do vínculo que possui com as famílias e comunidades (Delfini, Reis, 2012; Figueiredo, Onocko Campos, 2008), pois isto pode facilitar as intervenções do ponto de vista terapêutico. Nem sempre, porém, os profissionais da ESF possuem tecnologia para a resolução de algumas situações em saúde mental (Harada, Soares, 2010) e habilidade de comunicação para lidar com pessoas com transtornos mentais (Tófoli, Fortes, 2007).

Outro importante desafio para a saúde mental e a ESF está relacionado às situações de adoecimento provocadas pela miséria e pelas situações de violência enfrentadas por boa parte da população brasileira, e quanto estes fatores favorecem dificuldades afetivas, emocionais e relacionais (Figueiredo, Onocko Campos, 2008).

Deste modo, o apoio matricial em saúde mental é sugerido como uma estratégia para qualificar e ampliar a resolutividade das ações da ESF (Cossetin, Olschowsky, 2011; Brasil, 2003). Tal estratégia 
possibilita aos profissionais da saúde mental trocarem conhecimentos e práticas da área "psi" com os profissionais das equipes da ESF, dando-Ihes suporte para poderem compreender, intervir e acolher melhor as pessoas em sofrimento, bem como incluir, na sua prática, a dimensão subjetiva e social do ser humano, mediante uma escuta qualificada e sensível, facilitando a ampliação da clínica (Figueiredo, Onocko Campos, 2008).

Neste prisma, o MS propõe a saúde mental como uma das áreas de atuação do NASF, e o apoio matricial como uma estratégia importante na ação dessas equipes multiprofissionais de apoio à ESF (Brasil, 2009).

Conforme revelam estudos de avaliação, o apoio matricial, quando adequadamente implantado, ajuda na articulação da rede de serviços de saúde e na integração entre as equipes de saúde mental e da ESF (Onocko Campos et al., 2012, 2011).

Entretanto, o apoio matricial não pode ser considerado o único modelo a trazer efetividade aos cuidados de saúde mental na atenção primária (Mielke, Olschowshy, 2010). Portanto, outras estratégias, de acordo com as particularidades de cada território, devem ser desenvolvidas.

No tocante à produção bibliográfica recente sobre o tema, vale, ainda, destacar - apesar de não ser um documento oficial do Ministério da Saúde e nem um artigo científico - o "Guia prático de matriciamento em saúde mental" (Chiaverini et al., 2011), o qual foi financiado pelo Ministério da Saúde, mas redigido por um grupo independente de autores com experiência teórico-prática neste campo. Embora com ênfase no apoio matricial em saúde mental como estratégia de atuação da saúde mental na atenção primária, o guia abrange um conjunto de orientações bem mais amplas e com informações significativas para subsidiar os profissionais na troca de conhecimentos e na construção de estratégias de ação em saúde mental, com base na realidade dos usuários e do seu território de atuação.

\section{Desafios e potencialidades do apoio matricial e do NASF na saúde mental}

Vários artigos sobre saúde mental na APS destacaram obstáculos estruturais. Entre os principais pontos, constataram: sobrecarga dos profissionais e superlotação dos serviços, pela baixa cobertura de serviços de saúde mental e da ESF; carência e alta rotatividade dos profissionais na APS, que, possivelmente, podem ser atribuídas às condições de trabalho, à falta de perfil profissional e à forma de contratação; rigidez na agenda dos profissionais e cobrança por produtividade, faltando espaço para a reflexão sobre o processo de trabalho e o trabalho conjunto; e as mudanças nos modelos de organização dos serviços de uma gestão para outra (Delfini, Reis, 2012; Onocko Campos et al., 2011). Vale ressaltar que tais aspectos foram destacados em estudos que aconteceram em grandes centros urbanos.

Em relação à formação acadêmica dos profissionais da saúde mental e da ESF, existem limitações que comprometem a atuação destes na conjuntura da APS. As discussões e problematizações sobre o contexto social e subjetivo do processo saúde-doença requerem uma análise mais profunda, que vai além da técnica, e que, muitas vezes, passa à margem dos cursos de graduação da saúde (Onocko Campos, Gama, 2008).

Tal fato é confirmado em vários estudos (Onocko Campos et al., 2012, 2011; Cavalcante et al., 2011; Dimenstein et al., 2009b; Figueiredo, Onocko Campos, 2009), nos quais a formação dos profissionais da ESF é apontada como uma limitação para o atendimento dos usuários com sofrimento psíquico e a medicação é entendida, por vários profissionais, como a única possibilidade de ajuda para esses usuários ou como paliativo para o sofrimento provocado por questões sociais.

O apoio matricial em saúde mental configura-se como uma possibilidade para favorecer a ampliação da clínica na ESF. Entretanto, de acordo com a compreensão sobre o processo saúde-doença, do profissional da saúde mental responsável pela ação de apoio, a ampliação pode não acontecer e podese, inclusive, reforçar a lógica que se pretende superar (Dimenstein et al., 2009b).

Historicamente, os "clássicos" profissionais da saúde mental, psicólogos e psiquiatras, também na sua formação tradicional, com raras exceções, exercem uma clínica de amplitude limitada. Os profissionais da psicologia trazem forte influência da clínica tradicional e elitizada, com pouca ênfase nas questões sociais e de promoção da saúde e com privilégio dos aspectos individuais (Meira, Silva, 2011; 
Ferreira Neto, 2008). Já a psiquiatria contemporânea possui forte ênfase no aspecto biológico e nas medicações como forma exclusiva de tratamento (Onocko Campos, Gama, 2008).

Como aponta o MS (Brasil, 2009, p.12), "o conceito de apoio matricial tem uma dimensão sinérgica ao conceito de educação permanente". Esta ideia é corroborada em vários artigos (Meira, Silva, 2011; Bezerra et al., 2010; Bardan, Oliveira, 2007; Tófoli, Fortes, 2007), a sugerir os processos de educação permanente como uma estratégia para superação das limitações da graduação na saúde no enfrentamento da realidade cotidiana dos serviços. Estes processos de educação permanente estão ligados não somente aos processos educativos formais (residências, especializações, capacitações), mas, também, a processos de aprendizagem desenvolvidos no cotidiano no trabalho, mediante contato e troca de experiências com outros profissionais e usuários do SUS.

Na nossa ótica, a educação permanente se articula fortemente com os desafios na formação do trabalhador em saúde. Evidentemente, há dificuldades para se atuar em apoio matricial de maneira formativa, num cenário no qual a própria formação dos profissionais de saúde é ainda pouco dialógica e centrada dentro dos muros do próprio núcleo da sua categoria.

Conforme apontam Onocko Campos et al. (2012), a dificuldade de atuação do apoiador matricial parece estar atrelada à sensação de impotência, solidão e desamparo dos profissionais da atenção primária para lidarem com a complexidade da saúde mental.

Diversos artigos (Bezerra, Dimenstein, 2008; Soares, 2008; Bardan, Oliveira, 2007) mencionam a importância de o apoiador matricial em saúde mental deter alguns conhecimentos e habilidades para o bom desempenho dessa função, como por exemplo: dispor de habilidades de comunicação e de relacionamento interpessoal para lidar com as equipes da ESF e os usuários; ter disponibilidade para troca de conhecimento e experiência com outros profissionais; compreender a integralidade das redes assistenciais, dos serviços de saúde e a importância da atenção primária; exercer a visão ampliada do processo saúde-doença; e apresentar capacidade de estimular ações multidisciplinares de promoção e prevenção, considerando a complexidade dos transtornos mentais.

Diante dessa realidade, as discussões futuras, portanto, sobre a formação para o AM em saúde mental e a atuação neste tipo de organização do trabalho em saúde, necessitam obrigatoriamente enfatizar e aperfeiçoar este conjunto de competências.

No referente ao $\mathrm{NASF}^{5}$ (Brasil, 2009), este representa um avanço para as ações de saúde mental na ESF (Bezerra et al., 2010). Contudo, em face do grande número de equipes da ESF que um profissional de NASF deve apoiar - de oito a vinte -, corre-se o risco de reforçar a saúde mental como especialidade, praticamente inviabilizando o trabalho de prevenção e promoção em saúde mental (Böing, Crepaldi, 2010).

Além disso, o número excessivo de equipes dificultaria as ações interdisciplinares, que exigem uma presença e um contato marcantes da equipe do NASF no cotidiano de trabalho das equipes da ESF e na comunidade. Ou seja, apesar de o MS (Brasil, 2009) reconhecer a importância do vínculo entre as equipes para o trabalho do NASF e da ESF, esse vínculo é comprometido pela própria organização do processo de trabalho conforme determinado na portaria que cria os NASF (Brasil, 2008). Com isso, amplia-se o risco de as equipes do NASF se dedicarem, basicamente, à atenção curativa, reforçando o modelo de enfoque na doença e no sintoma (Böing, Crepaldi, 2010). 
Alguns dos artigos analisados e publicações do MS trazem orientações e sugestões sobre as práticas de apoio matricial em saúde mental. Estas configuram um conjunto de dispositivos e ferramentas, entre os quais podemos destacar: elaboração de projeto terapêutico singular; consulta e visita domiciliar conjunta; uso de recurso de comunicação pessoal (celular, e-mail) entre profissionais, para facilitar o contato em situações de urgência. Foge ao escopo deste trabalho detalhar esses itens, mas, inegavelmente, eles complementam as habilidades e conhecimentos relativos ao AM em SM descritos anteriormente.

Por fim, vale acrescentar que o papel dos agentes comunitários de saúde foi reconhecido como extremamente importante nas ações de apoio matricial (Onocko Campos et al., 2011). Por conta da sua aproximação e vinculação maior com a realidade social e a população atendida, os ACS trazem grande potencialidade às ações de saúde mental. Seu papel no AM em SM, entretanto, ainda precisa ser melhor explorado em estudos futuros.

\section{Considerações finais}

Segundo demonstram os artigos científicos e os documentos analisados, a lógica de organização do trabalho em saúde a partir das diretrizes do apoio matricial requer a valorização das relações verificadas no cotidiano do trabalho em saúde e dos vínculos afetivos entre profissionais e usuários, entre os próprios profissionais, e entre estes e os gestores. Entretanto, como reforça a produção analisada, para isso acontecer, o dia a dia dos serviços de saúde deve ser permeado por espaços de reflexão sobre as práticas e os saberes desenvolvidos pelos profissionais, a partir do contato com a realidade da população do seu território de abrangência. Este arranjo exige, de profissionais e gestores, algo além da competência técnica. Ele enfatiza a necessidade de uma competência relacional, de uma atitude de abertura para o diálogo e para o saber construído coletivamente, sem, no entanto, descartar o conhecimento nuclear de cada categoria profissional. Neste âmbito, os processos de cogestão e de educação permanente foram apontados como facilitadores dessa mudança organizacional.

Para o conceito de apoio matricial se transformar em realidade, ele deve ser fruto de um trabalho coletivo de pessoas que se unem na perspectiva de transformar a fragmentação e alienação existente no processo de trabalho em saúde, com vistas a ampliar a resolutividade e a qualidade dos serviços oferecidos à população brasileira e compor, de forma harmônica, um novo modelo de atenção à saúde em nosso país.

Porém, a lógica hierárquica, burocrática e centrada em procedimentos coexiste com as diretrizes do apoio matricial. Estas diretrizes impõem que os sujeitos envolvidos neste processo passem a conviver com a estranheza e a complexidade presentes nessas formas distintas de organização do trabalho em saúde.

Nesse sentido, o NASF foi apontado como um avanço importante na implantação do apoio matricial em saúde mental na atenção primária. Contudo, parece que, nele, as contradições dessas lógicas distintas tornam-se ainda mais evidentes, sobretudo pelo número ${ }^{6}$ de equipes da ESF que cada NASF tem sob sua responsabilidade (Böing, Crepaldi, 2010). Ademais, as ações de saúde mental na ESF não devem ficar circunscritas ao modelo do apoio matricial.

As diferentes experiências de apoio matricial em saúde mental na ESF trazem muitos pontos relevantes passíveis de contribuir com outros municípios que estejam avançando na integralidade das ações da ESF. Entre estes, podemos destacar: o imperativo de qualificar os profissionais da ESF para lidar com o

${ }^{6}$ É importante destacar que, na data de aprovação deste artigo pelos avaliadores da revista, o NASF ainda era regido pela portaria da sua criação (Brasil, 2008). 
sofrimento humano; a necessidade de maior interlocução entre as várias categorias profissionais para ampliar a resolutividade das ações; a urgência de abertura para o diálogo e para a troca de experiências entre profissionais e entre estes e os gestores; a relevância dos vínculos afetivos entre os profissionais, para facilitar essa abertura; e a necessidade de o apoiador matricial ter uma compreensão integral da rede de saúde e da complexidade dos transtornos mentais, além do desenvolvimento de um conjunto de competências voltadas para o apoio matricial em saúde mental que ainda estão em construção.

A ideia de construção, aliás, é premente ao analisarmos estes documentos e artigos. Evidentemente, este é um processo dinâmico, inédito e aberto à cooperação futura. É essencial, assim, compreendermos que uma delimitação mais clara desse campo ainda está por vir, embora seus contornos possam ser - como esta revisão indica - cada vez mais distintos. Portanto, novos aportes teórico-práticos e estudos avaliativos são necessários e bem-vindos para o aperfeiçoamento do apoio matricial em saúde mental na atenção primária, como componente do modelo de atenção à saúde.

\section{Colaboradores}

Íris Guilherme Bonfim responsabilizou-se pela elaboração do artigo, com apoio na discussão teórico-conceitual sob a responsabilidade de Cezar Wagner de Lima Góis; Evelyne Nunes Ervedosa Bastos e Luis Fernando Tófoli responsabilizaram-se pela revisão crítica do manuscrito.

\section{Referências}

ARONA, E.C. Implantação do matriciamento nos serviços de saúde de Capivari. Saude Soc., v.18, n.1, p.26-36, 2009.

BARBAN, E.G.; OLIVEIRA, A.A. O modelo de assistência da equipe matricial de saúde mental no programa saúde da família do município de São José do Rio Preto (Capacitação e educação permanente aos profissionais de saúde na atenção básica). Arq. Cienc. Saude, v.14, n.1, p.52-63, 2007.

BEZERRA, E.; DIMENSTEIN, M. Os CAPS e o trabalho em rede: tecendo o apoio matricial na atenção básica. Psicol. Cienc. Prof., v.28, n.3, p.632-45, 2008.

BEZERRA, R.S.S. et al. Arranjo matricial e o desafio da interdisciplinaridade na atenção básica: a experiência do NASF em Camaragibe/PE. Divulg. Saude Debate, n.46, p.51-59, 2010.

BÖING, E.; CREPALDI, M.A. O psicólogo na atenção básica: uma incursão pelas políticas públicas de saúde brasileiras. Psicol. Cienc. Prof., v.30, n.3, p.634-49, 2010.

BONFIM, I.G. Apoio Matricial em Saúde Mental na Estratégia de Saúde da Família em Fortaleza: ouro que não boia. 2012. Dissertação (Mestrado em Psicologia) - Centro de Humanidades, Universidade Federal do Ceará, Fortaleza, 2012.

Apoio Matricial em Saúde Mental na Atenção Básica: uma análise da produção científica e das experiências. 2009. Monografia (Especialização) - Centro de Ciências da Saúde, Universidade Estadual do Ceará, Fortaleza. 2009.

BRASIL. Ministério da Saúde. Secretaria de Atenção à Saúde. DAPES. Coordenação Geral de Saúde Mental, Álcool e Outras Drogas. Saúde Mental no SUS: as novas fronteiras da Reforma Psiquiátrica. Brasília: MS, 2011. 
BRASIL. Ministério da Saúde. Secretaria de Atenção à Saúde. Departamento de Atenção Básica e Departamento de Ações Programáticas Estratégicas. Diretrizes NASF - Núcleo de Apoio à Saúde da Família. Brasília: MS, 2009.

Ministério da Saúde. Portaria 154, de 24 de janeiro de 2008. Dispõe sobre a criação dos Núcleos de Apoio à Saúde da Família. Disponível em: <http:// www.sbp.com.br/pdfs/portaria_154.pdf>. Acesso em: 1 jul. 2012.

Ministério da Saúde. Secretaria de Atenção à Saúde. Relatório de Gestão 2003-2006: saúde mental no SUS: acesso ao tratamento e mudança do modelo de atenção. Brasília: MS, 2007.

Ministério da Saúde. Secretaria de Atenção à Saúde. Departamento de Ações Programáticas Estratégicas. Coordenação Geral de Saúde Mental. Reforma Psiquiátrica e a Política de Saúde Mental no Brasil. Brasília: MS, 2005.

. Ministério da Saúde. Secretaria Executiva. Núcleo Técnico da Política Nacional de Humanização. HumanizaSUS: equipe de referência e apoio matricial. Brasília: MS, 2004a.

- Ministério da Saúde. Secretaria de Atenção à Saúde. Departamento de Ações Programáticas Estratégicas. Saúde mental no SUS: os centros de atenção psicossocial. Brasília: MS, 2004b.

Ministério da Saúde. Secretaria-Executiva. Núcleo Técnico da Política Nacional de Humanização. HumanizaSUs: política nacional de humanização: documento base para gestores e trabalhadores do SUS. Brasília: MS, 2004c.

. Ministério da Saúde. Coordenação de Saúde Mental e Coordenação de Gestão da Atenção Básica. Saúde Mental e Atenção Básica: o vínculo e o diálogo necessários. Brasília: MS, 2003

CAMPOS, G.W.S. Equipes de referência e apoio especializado matricial: um ensaio sobre a reorganização do trabalho em saúde. Cienc. Saude Colet., v.4, n.2, p.393-403, 1999.

O anti-Taylor: sobre a invenção de um método para co-governar instituições de saúde produzindo liberdade e compromisso. Cad. Saude Publica, v.14, n.4, p.863-70, 1998.

Modelos assistenciais e Unidades Básicas de Saúde: elementos para debate. In: CAMPOS, G.W.S.; MERHY, E.E.; NUNES, E.D. (Orgs.). Planejamento sem norma. 2.ed. São Paulo: Hucitec, 1989. p.53-60.

CAMPOS, G.W.S.; CUNHA, G.T. Apoio matricial e atenção primária em saúde. Saude Soc., v.20, n.4, p.961-70, 2011.

CAMPOS, G.W.S.; DOMITTI, A.C. Apoio matricial e equipe de referência: uma metodologia para gestão do trabalho interdisciplinar em saúde. Cad. Saude Publica, v.23, n.2, p.399-407, 2007.

CAMPOS, G.W.S.; RATES, S.M.M. Segredos e impasses na gestão de um hospital público. Rev. Med. Minas Gerais, v.18, n.4, p.279-83, 2008.

CARNeIRO, A. C. et al. Saúde Mental e Atenção Primária: uma experiência com agentes comunitários de saúde em Salvador-BA. Rev. Bras. Prom. Saude, v.22, n.4, p.264-71, 2009.

CAVALCANTE, C.M. et al. Desafios do cuidado em saúde mental na Estratégia Saúde da Família. Rev. Bras. Prom. Saude, v.24, n.2, p.102-8, 2011.

CHIAVERINI, D.H. et al. Guia prático de matriciamento em Saúde Mental. Brasília: Ministério da Saúde, Centro de Estudos e Pesquisa em Saúde Coletiva, 2011. 
COSSETIN, A.; OLSCHOWSKY, A. Avaliação das ações em saúde mental na Estratégia de Saúde da Família: necessidades e potencialidades. Rev. Gaucha Enferm., v.32, n.3, p.495-501, 2011.

DELFINI, P.S.S.; REIS, A.O.A. Articulação entre serviços públicos de saúde nos cuidados voltados à saúde mental infantojuvenil. Cad. Saude Publica, v.28, n.2, p.357-66, 2012

DELFINI, P.S.S. et al. Parceria entre CAPS e PSF: o desafio da construção de um novo saber. Cienc. Saude Colet., v.14, n.1, p.1483-92, 2009.

DIMENSTEIN, M. et al. O apoio matricial em Unidades de Saúde da Família: experimentando inovações em saúde mental. Saude Soc., v.18, n.1, p.63-74, 2009a.

DIMENSTEIN, M.; GALVÃO, V.M.; SEVERO, A.K.S. O Apoio Matricial na perspectiva de coordenadoras de Equipes de Saúde da Família. Pesqui. Prat. Psicossoc., v.4, n.1, p.37-48, 2009b.

FERREIRA NETO, J.L. Psicologia e Saúde Mental: três momentos de uma história. Saude Debate, v.32, n. 78-80, p.18-26, 2008.

FIGUEIREDO, M.D.; ONOCKO CAMPOS, R. Saúde Mental na Atenção Básica à Saúde de Campinas, SP: uma rede ou um emaranhado? Cienc. Saude Colet., v.14, n.1, p.129-38, 2009

Saúde Mental e Atenção Básica à Saúde: o apoio matricial na construção de uma rede multicêntrica. Saude Debate, v.32, n.78-80, p.143-9, 2008.

GIL, A.C. Métodos e técnicas de pesquisa social. 5.ed. São Paulo: Atlas, 1999.

GOIS, C.W.L. Saúde comunitária: pensar e fazer. São Paulo: Aderaldo \& Rothschild, 2008

HARADA, O.L.; SOARES, M.H. A percepção do agente comunitário de saúde para identificar a depressão. Rev. Eletr. Saude Mental Álcool Drog., v.6, n.2, p.315-36, 2010. Disponível em: <http://pepsic.bvsalud.org/pdf/smad/v6n2/6.pdf>. Acesso em: 7 ago. 2012

LEOPARDI, M.T. Metodologia da pesquisa na saúde. Florianópolis: UFSC, PósGraduação em Enfermagem, 2002.

MEIRA, M.A.; SILVA, M.O. Atuação da Psicologia na Estratégia Saúde da Família: a experiência de um psicólogo em uma residência multiprofissional. Rev. Bras. Cienc. Saude, v.15, n.3, p.369-76, 2011.

MENDES, E.V. As redes de atenção à saúde. Cienc. Saude Colet., v.15, n.5, p.2297-305, 2010.

MIELKE, F.B.; OLCHOWSKY, A. Saúde mental na Estratégia Saúde da Família: a avaliação de apoio matricial. Rev. Bras. Enferm., v.63, n.6, p.900-7, 2010.

OLIVEIRA, G.N. Apoio Matricial como tecnologia de gestão e articulação em rede. In: CAMPOS, G.W.S; GERREIRO, A.V.P. (Orgs.). Manual de práticas da Atenção Básica: saúde ampliada e compartilhada. São Paulo: Hucitec, 2008. p.263-72.

ONOCKO CAMPOS, R. et al. Avaliação de estratégias inovadoras na organização da Atenção Primária à Saúde. Rev. Saude Publica, v.46, n.1, p.43-50, 2012.

Saúde mental na atenção primária à saúde: estudo avaliativo em uma grande $\overline{\text { cidade }}$ brasileira. Cienc. Saude Colet., v.16, n.12, p.4643-52, 2011.

ONOCKO CAMPOS, R.; GAMA, C. Saúde Mental na Atenção Básica. In: CAMPOS, G.W.S; GERREIRO, A.V.P (Orgs.). Manual de práticas em Atenção Básica: saúde ampliada e compartilhada. São Paulo: Hucitec, 2008. p.210-36. 
ORGANIZAÇÃO MUNDIAL DA SAÚDE - OMS. Cuidados inovadores para condições crônicas: componentes estruturais de ação: relatório mundial. Brasília: OMS, 2003.

ORGANIZAÇÃO PANAMERICANA DE SAÚDE - OPAS. Declaração de Alma-Ata. Conferência Internacional sobre Cuidados Primários de Saúde. Alma-Ata: OPAS, 1978.

PAIM, I.S. A reforma sanitária e os modelos assistenciais. In: ROUQUAYROL, M.Z. (Org.). Epidemiologia \& Saúde. 4.ed. Rio de Janeiro: MEDSI, 1994. p.455-66.

PRESTES, L.I.N. et al. Apoio Matricial: um caminho de fortalecimento das redes de atenção à saúde em Palmas-TO. Rev. Bras. Cienc. Saude, v.15, n.2, p.215-8, 2011.

SOARES, M.H. A inserção do enfermeiro psiquiátrico na equipe de apoio matricial em saúde mental. SMAD - Rev. Eletr. Saude Mental Álc. Drog., v.4, n.2, 2008. Disponível em: <http://pepsic.bvsalud.org/pdf/smad/v4n2/v4n2a06.pdf >. Acesso em: 6 ago. 2012.

SOUSA, F.S.P. et al. Tecendo a rede assistencial em saúde mental com a ferramenta matricial. Physis, v.21, n.4, p.1579-99, 2011.

TEIXEIRA, C.F. A mudança do modelo de atenção à saúde no SUS: desatando nós, criando laços. In: TEIXEIRA, C.F;; SOLLA, J.P. (Orgs.). Modelo de atenção à saúde: promoção, vigilância e saúde da família. Salvador: EDUFBA, 2006. p.19-58.

TÓFOLI, L.F.; FORTES, S. Apoio Matricial de Saúde Mental na atenção primária no Município de Sobral-CE: o relato de uma experiência. SANARE - Rev. Pol. Pub. Sobral/CE, v.6, n.2, p.34-42, 2007.

VIEIRA FILHO, N.G.; ROSA, M.D. Clínica psicossocial: articulando saúde mental e a estratégia saúde da família. Mental, v.9, n.16, p.303-26, 2011.

BONFIM, I.G. et al. Apoyo matricial en salud mental en la atención primaria a la salud: un análisis de la producción científica y documental. Interface (Botucatu), v.17, n.45, p.287-300, abr./jun. 2013.

El reto de hacer efectiva la integralidad de la atención en el sistema gubernamental de salud brasileño (SUS) favorece la discusión acerca del apoyo matricial (AM) para la Estrategia de Salud Familiar (ESF) y refuerza la necesidad de más estudios sobre el tema. En este artículo se analiza críticamente el apoyo matricial por medio de las publicaciones en revistas científicas y documentos del Ministerio de Salud de Brasil, especialmente los enfocados en el apoyo matricial de salud mental (SM) en la atención primaria. El material estudiado señala la importancia de la coordinación entre la SM y la atención primaria, pero también indica que el AM en la SM forma parte de un modelo de atención a la salud aún en construcción, y que son indispensables nuevas contribuciones teórico-prácticas y evaluativas para su perfeccionamiento.

Palabras clave: Apoyo matricial. Salud mental. Atención primaria. Estrategia de salud familiar.

Recebido em 22/08/12. Aprovado em 10/03/13 